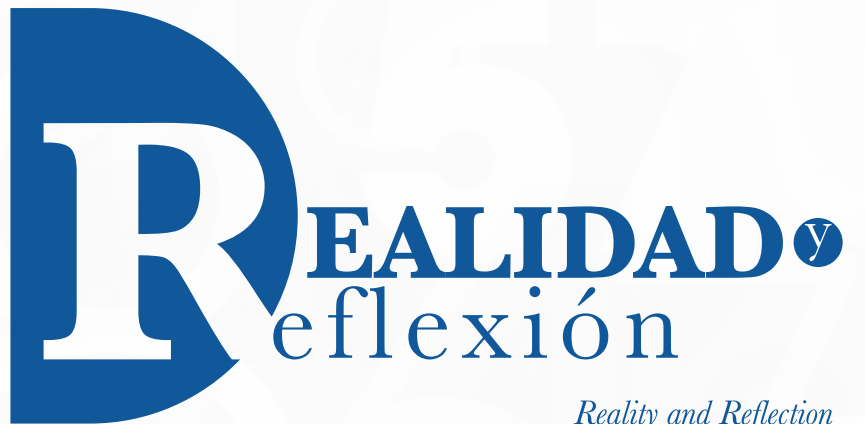

ISSN 1992-6510

Año 21, N 53, San Salvador, El Salvador, Centroamérica. Revista Semestral Enero-Junio 2021

Year 21, N 53, San Salvador, El Salvador, Central America. Semestral Journal January-June 2020

\title{
La resolución de problemas en la formación inicial del profesorado de Primaria: una experiencia de aula
}

\author{
Problem solving in prospective primary teachers: \\ a classroom experience
}

Luis Carlos Contreras González Licenciado en Matemáticas por la Universidad de Sevilla y Doctor en Psicopedagogía por la Universidad de Huelva. Catedrático de Didáctica de la Matemática en la Universidad de Huelva, España lcarlos@uhu.es

Recibido: 25 de mayo de 2020 Aceptado: 24 de agosto de 2020 DOI: $10.5377 /$ ryr.v53i53.10896

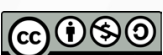




\section{RESUMEN}

En este artículo reflexionamos acerca del papel de la resolución de problemas en la formación inicial de profesores de Educación Primaria. Tras retomar algunas aportaciones relevantes en el ámbito de la resolución de problemas en la educación matemática, nos centramos en la doble vertiente de plantear y de resolver problemas, analizando la relevancia que esa doble componente tiene, tanto en la educación obligatoria, como en la formación inicial de profesores. El último epígrafe del artículo comienza con una contextualización de la formación en educación matemática de los futuros maestros de Primaria en la Universidad de Huelva, para cerrar detallando el desarrollo de una experiencia de aula que ejemplifica cómo trabajamos con los futuros maestros "aprender y aprender a enseñar a plantear y resolver problemas".

Palabras clave: Formación Inicial de Profesores de Primaria, Planteamiento de Problemas, Resolución de Problemas, Educación Matemática

\section{ABSTRACT}

In this article we reflect on the role of problem solving in the prospective primary teachers' training. After taking up some relevant contributions in the field of problem solving in mathematics education, we focus on the double aspect of problems posing and problems solving, analyzing the relevance that this double component has, both in compulsory education and in prospective primary teachers' training. The last part of the work begins with a contextualization of the training in mathematics education of future primary teachers at the University of Huelva, to close detailing the development of a classroom experience that exemplifies how we work with future teachers "learn and learn to teach how to pose and solve problems".

Keywords: Prospective Primary Teacher's Training, Problem Posing, Problem Solving, Mathematics Education. 


\section{Introducción}

La resolución de problemas ha tenido un papel destacado, tanto en las matemáticas, como en la enseñanza y el aprendizaje de las matemáticas, desde la segunda mitad del siglo XX; la mayoría de los autores sitúan el comienzo en los trabajos de Polya (1945), centrado en su interés en cómo resolver los problemas de aprendizaje de sus estudiantes. Desde entonces, ha ocupado y ocupa un papel relevante planes de estudio de la enseñanza obligatoria en todo el mundo, en los que, tanto el énfasis en la enseñanza de la resolución de problemas, per se (enseñar a resolver problemas), como de la enseñanza de las matemáticas a través de la resolución de problemas (enseñar vía resolución de problemas), es el hilo conductor y organizador curricular. En paralelo, ha sido un foco de interés para los investigadores de educación matemática, basta analizar el protagonismo que ha tenido en todas las conferencias de ICME, desde 1969 hasta la de Hamburgo, en 2016, donde la resolución de problemas matemáticos ocupó el centro del Grupo de Trabajo 19: Resolución de problemas en la educación matemática (Liljedah, Santos-Trigo, Malaspina y Bruder, 2016). En los momentos en que escribo estas líneas se ha anunciado el aplazamiento, para 2021, del ICME 14, previsto para julio de 2020, en el que el TSG 17 iba a versar sobre problem posing and solving in mathematics education.

Lester y Kehle (2003) caracterizan la resolución de problemas matemáticos como una actividad en la que los estudiantes se implican y realizan un importante elenco de acciones cognitivas que conllevan el acceso y el uso de conocimientos y experiencias previas. Además, deben coordinar diferentes registros de representación, encontrar patrones y poner a prueba su capacidad de intuir e inferir. Como señalan Verschaffel, Greer y De Corte (2000), hay suficientes evidencias empíricas que nos animan al desarrollo de métodos de aprendizaje orientados a procesos que enfaticen la resolución de problemas matemáticos, dado que se muestra como un aspecto de especial dificultad para los estudiantes de Primaria.

Como señala Kramarski (2009), en los estándares de educación matemática, desde un enfoque del aprendizaje a lo largo de la vida, se apunta a la resolución de problemas como el eje sobre el que vertebrar, no solo la educación obligatoria, sino también la formación de profesores de matemáticas (NCTM, 2000; INECSE, 2003). Desde esta visión de los estándares, la educación matemática y la formación del profesorado debería centrarse en la resolución de problemas, el razonamiento matemático y la comunicación como integradores curriculares. Desde esa perspectiva, los profesores han de enfrentarse a un desafío que supone enseñar matemáticas guiando a sus estudiantes a obtener ideas matemáticamente potentes. Como señalan Hill, Rowan y Ball (2005) o Putnam y Borko (2000), es preciso orientar la enseñanza de las matemáticas hacia procesos en los que los estudiantes se enfrenten a retos fomentando la construcción del conocimiento a través del aprendizaje autorregulado en la medida en que son participantes activos en su propio proceso de aprendizaje (Zimmerman, 2000).

No obstante, como señalan estos mismos autores (Hill, et. al., 2005; Putnam y Borko, 2000), los maestros de Educación Primara que salen de nuestros centros de formación inicial siguen sin estar preparados para enseñar matemáticas de esta manera prevista los estándares, fundamentalmente porque la mayoría 
de ellos no han aprendido las matemáticas de esta manera, sino más bien desde el enfoque instrumental tradicional, donde el contenido matemático se asemeja a un conjunto de hechos, conceptos y principios, muchas veces sin vínculos entre sí, no relacionados, para ser aplicados en listados de ejercicios de aplicación. Los espacios para pensar y razonar, comunicar, argumentar, modelizar, representar, plantear y resolver problemas y utilizar el lenguaje simbólico, formal y técnico sobre situaciones para las que no hay una respuesta inmediata no han caracterizado sus aprendizajes. Los estudios muestran que estas experiencias anteriores suelen generar unas creencias sobre la matemática y sobres su enseñanza y aprendizaje que, a menudo sirven como una lente a través de la cual los maestros filtran el nuevo conocimiento pedagógico que se les ha enseñado. Esos mismos estudios señalan que es esencial que los formadores de profesores tengan en cuenta la existencia de estas creencias al momento de formar profesores (Pajares, 1992; Kramarski y Michalsky, 2009; Richardson, 1996; Thompson, 1992).

Por otro lado, en la cadena de transmisión/construcción del conocimiento matemático ocupa un papel relevante el libro de texto que utiliza el profesor.Jäder, Lihtner y Sindenvall (2019) llaman nuestra atención en este sentido, señalando al libro de texto como uno de los factores influyentes en el resultado de la educación matemática y nos recuerdan que ya Schoenfeld (1985) afirmaba que, para desarrollar una competencia de resolución de problemas, uno necesita trabajar con problemas, es decir, necesita disponer de problemas, bien de elaboración propia, bien presentes en algún recurso como el libro de texto o en plataforma digitales. Sin embargo, su estudio muestra que la gran mayoría de las tareas en todos los libros de texto analizados eran tareas de reproducción, en las que los alumnos disponían de los recursos suficientes para resolver sin que ello supusiera un reto para ellos. Esto nos pone de relieve la necesidad de una selección o elaboración cuidadosa (por parte de los maestros, pero también de los estudiantes) de recursos para crear oportunidades para desarrollar la competencia para resolver problemas en este sentido. Esto último nos sitúa ante una doble consideración de la resolución de problemas: plantearlos y resolverlos.

\section{Problem solving y problem posing”}

Singer, Ellerton, Cai y Leung (2011) aluden de forma indirecta al libro de texto cuando afirman que, cuando se resuelven problemas en el aula, estos suelen estar planteados por alguien externo a ella. Hay varios mensajes en esta afirmación que conviene resaltar. En primer lugar, parecen indicarnos que, en el mejor de los casos, cuando se resuelven problemas que emanan de fuentes externas al aula, puede no existir una intencionalidad predeterminada por parte del profesor en su selección. Dicho en positivo, cuando un profesor plantea un problema (lo elija o lo elabore) ha de hacerlo con una finalidad predefinida; para mejorar la capacidad de comprensión de los enunciados por parte de sus estudiantes, para favorecer los cambios de registro de la información, para potenciar la capacidad de organizar la información y otros elementos vinculados a la planificación, para desarrollar otras herramientas heurísticas, ... Parece obvio que, si el profesor decide que sus estudiantes resuelvan determinados problemas, con una finalidad predeterminada, será más eficaz elaborar el problema para responder a esa finalidad, que encontrar uno que cumpla tal condición en una fuente externa. En segundo 
lugar, la afirmación de estos autores también parece recordarnos que tan importante es que nuestros alumnos resuelvan problemas, como que sepan plantearlos. Coincidimos con Singer et al. (2011) cuando afirman que tanto profesores como estudiantes están poco habituados en la tarea de plantear problemas. Dediquemos unas líneas a reflexionar sobre la importancia saber plantear problemas.

Durante las últimas dos décadas, la investigación en educación matemática ha producido un importante número de artículos que indagan en la relación entre la plantear y resolver problemas (Christou, Mousoulides, Pittalis, Pitta-Pantazi y Sriraman, 2005; K1lıç, 2013, Lowrie, 2002; Luo, 2009; Tichá y Hošpesová, 2009), siendo concluyentes en la ayuda que supone plantear problemas en el proceso de formación en resolución de problemas.

Una de las claves de la resolución de problemas es ser capaz de analizar las características del resultado. El proceso de resolución se antoja incompleto si el resolutor no se hace preguntas acerca de qué ocurriría si cambiaran determinadas condiciones iniciales. Cuando entrenamos a los resolutores (sean estudiantes de primaria o estudiantes para profesor de Primaria) en el arte de hacerse este tipo de preguntas estamos abriendo la puerta al planteamiento de nuevos problemas. Como señalan Singer et al. (2011), cuando se entrena de forma sistemática en la transformación de problemas, cambiando representaciones, operaciones o condiciones, se sitúa al resolutor ante la posibilidad de comparar varios problemas evaluando similitudes y diferencias, incrementando sus capacidades de apreciar lo esencial entre lo auxiliar y ayudándoles a comprender la estructura que los hace semejantes. Cuando se aborda este tipo de tareas, se promueve que los estudiantes agudicen su capacidad de observar, experimentar, variando datos o contexto, comparar los resultados y generar, así, nuevos problemas (Singer y Voica, 2008). Ver el final de cada problema como el principio de otro hace que el planteamiento y resolución de problemas se convierta en un ciclo dinámico y constante en el que, a diferencia de planteamientos convencionales de ejercitación repetitiva, el producto final pierde importancia frente a la reflexión acerca de los métodos y estrategias que se utilizan para lograrlo (Stanic y Kilpatrick, 1988; Karimi, Shahvarani y Haghverdi, 2019).

Dicen Christou, et al. (2005, p. 149), que "plantear problemas es un aspecto importante de las matemáticas puras y aplicadas y una parte integral de los ciclos de modelado que requieren la idealización matemática de fenómenos del mundo real". Podría decirse que plantear problemas es la componente esencial de la resolución de problemas, sin embargo, la investigación en educación matemática no le dedicado tanta atención como a la resolución de problemas. Como señala Ellerton (2013, pp. 100-101): “durante demasiado tiempo, el objetivo prioritario ha sido la resolución exitosa de problemas; ha llegado el momento de plantear problemas para que se les otorgue un lugar destacado en los currículos y aulas de matemáticas”. Pensamos que esto es extensible a los currículos de formación de profesores de matemáticas; los futuros profesores, que no han sido entrenados en plantear problemas, cuando eran estudiantes, requieren una atención especial en este sentido. Pueden parecer nuevos estos planteamientos, sin embargo, ya Kilpatrick (1987, p. 123) señalaba que "la formulación de problemas debía ser vista no solo como un objetivo de instrucción sino también como un medio de instrucción". 


\section{La formación inicial de profesores de Primaria en España}

Cuando España adaptó sus títulos universitarios al Espacio Europeo de Educación Superior se produjo un cambio importante en la formación de profesores de Primaria. Estos cambios son especialmente visibles en los planes de estudio actuales, en los que los cambios más relevantes se centran en su estructuración en cuatro años de estudios, en vez de tres como antes de la adaptación, y en los que el carácter generalista a la formación de Maestros (ahora graduados en Educación Primaria) es todavía más patente.

Si ponemos el foco en los contenidos de la formación, los nuevos planes son sensibles a las aportaciones de la comunidad de educadores matemáticos a lo largo de la segunda mitad del siglo XX, enfatizando el carácter profesional del conocimiento de un Maestro, en cuanto al contenido matemático se refiere. Si se hace un análisis retrospectivo de esta formación, el contenido ha mantenido una dualidad desde las componentes disciplinar y didáctica; frente a esa tendencia, ahora es cada vez más frecuente ver cómo las universidades avanzan hacia una integración, que en nuestro país comenzó a ponerse de manifiesto con el título de las materias ("Matemáticas y su Didáctica") que formaban parte de los planes y manuales formativos de finales del siglo pasado. Así, la didáctica de la matemática pasó de ser el epílogo que los libros y programas de la segunda mitad del siglo XX presentaban tras cada contenido matemático específico, elaborados desde una perspectiva formal y academicista inspirada en una visión bourbakista de la Matemática, a los programas actuales que contemplan la integración de los dos dominios, el conocimiento de Matemáticas y el conocimiento derivado de la investigación en Educación Matemática.

Como hemos puesto de relieve en Carrillo y Contreras (2020), la investigación sobre el conocimiento del profesor, realizada en las tres últimas décadas, ha impulsado progresivamente esta visión más integrada del conocimiento del Maestro en el ámbito de las matemáticas, que hoy posee una perspectiva más profesional del mismo. Así, los programas de formación de Maestros de Educación Primaria en España abordan el conocimiento matemático no desde su perspectiva formal y academicista, sino como objeto de enseñanza y aprendizaje, como puede verse en diversos materiales coordinados por formadores de Maestros de diversas universidades españolas, que son utilizados como manuales de referencia en la formación inicial (Castro, 2001; Chamorro, 2003; Godino, 2004; Segovia y Rico, 2011; Flores y Rico, 2015; Carillo, Contreras, Climent, Montes, Escudero-Ávila y Flores-Medrano, 2016), o como libros específicos de enseñanza y aprendizaje de núcleos temáticos concretos (colección Matemáticas, Cultura y Aprendizaje de Ed. Síntesis).

Hoy, en las universidades españolas, la formación se articula, como he indicado antes, en cuatro años académicos, los dos primeros con una formación de corte teórico-práctica, que se realiza en las universidades, y los dos últimos con una formación, compartida entre la universidad y los centros educativos de Educación Primaria (Practicum), por un total de 480 horas lectivas. La formación, 
que consta de 240 créditos, se completa en esos cuatro cursos académicos con contenidos de otras didácticas específicas (Didáctica de las Ciencias Experimentales, Didácticas de las Ciencias Sociales, Didáctica de la Expresión Plástica, Musical y Corporal y Didáctica de la Lengua y la Literatura) con peso crediticio muy similar al que acabamos de detallar para la Didáctica de la Matemática y que conforman un total de 100 créditos de Formación Obligatoria, las materias de la Formación Básica (60 créditos de materias relacionadas con la Psicología Evolutiva y de la Educación, la Didáctica y Organización Escolar, la Sociología o la Investigación Educativa), 30 créditos de Materias Optativas, que dependen de cada universidad, 44 créditos de Prácticas Externas en centro educativos y 6 créditos del Trabajo de fin de Grado.

Además de dos semestres en los que se desarrolla el Practicum, los contenidos teórico-prácticos de la formación en Educación Matemática a que antes hemos hecho referencia suelen ocupar entre tres y cuatro de los seis semestres que dura esa parte de la formación (tres cursos académicos), según las universidades, y tienen como referencia los contenidos matemáticos de la Educación Primaria, recogidos en la materia genérica denominada Enseñanza y Aprendizaje de las Matemáticas, que se desarrollan en asignaturas como ${ }^{1}$ "Introducción a la enseñanza y aprendizaje de las matemáticas en Educación Primaria”, "Números y Operaciones”, "La construcción del lenguaje matemático, magnitudes y medida y tratamiento de la información, azar y probabilidad” y "Las formas, las figuras y sus propiedades".

Entre los objetivos de estas asignaturas, extraídos literalmente de los planes de estudio, se encuentran los siguientes ${ }^{2}$ : "Conocer los principios fundamentales relativos a los contenidos de las matemáticas escolares, reconociendo relaciones entre ellos, enunciar y resolver problemas matemáticos relativos a los contenidos de las matemáticas escolares en diversas situaciones y relacionándolo con consideraciones sobre su aprendizaje, analizar, razonar y comunicar eficazmente argumentaciones matemáticas relativas a los contenidos matemáticos escolares, conocer y relacionar los elementos del currículo respecto a la enseñanza de los contenidos matemáticos en Educación Primaria (objetivos, contenidos, recomendaciones metodológicas, evaluación), describiéndolos críticamente y relacionándolos con propuestas de enseñanza, cuestionarse y argumentar qué contenidos matemáticos enseñar en primaria, para qué y cómo, superando visiones simplificadoras e integrando consideraciones del currículo oficial y de la investigación sobre el aprendizaje de los contenidos, identificar distintas situaciones, fenómenos y representaciones relativos a los contenidos de las matemáticas escolares, describiendo sus diferencias en cuanto a los contenidos como objeto de enseñanza-aprendizaje, analizar críticamente propuestas, materiales y recursos para la enseñanza de los contenidos matemáticos de la Educación Primaria, argumentando posibles beneficios y obstáculos para el aprendizaje, diseñar materiales y actividades para

\footnotetext{
1 Específicamente en la Universidad de Huelva.
}

2 La cursiva en este último párrafo se efectúa para resaltar aquellos contenidos directamente relacionados con la resolución de problemas. 
la enseñanza de los contenidos matemáticos de Educación Primaria y la evaluación de su aprendizaje, considerando cuestiones sobre el contenido, el aprendizaje y la enseñanza y haciendo explícitas las decisiones tomadas y sus razones, justificar el posible proceso de pensamiento matemático de alumnos manifestados en sus producciones, relativos a los contenidos de las matemáticas escolares, considerando nociones sobre el aprendizaje de dichos contenidos, identificar modos de comunicación y estrategias de interacción en el aula de matemáticas, argumentando sus implicaciones para el aprendizaje, valorar el análisis de situaciones de enseñanza-aprendizaje matemáticos como vía de aprendizaje como profesor y valorar el trabajo con compañeros como vía de aprendizaje como profesor".

El planteamiento y resolución de problemas, como se ha visto, es transversal en el plan de estudios de nuestra universidad y la existencia de una materia, en el primer curso (segundo semestre) dedicada exclusivamente a la finalidad de enseñar a plantear y resolver problemas, puede decirse que es casi exclusivo de la misma. La idea de situarla en el primer curso era justamente para iniciar el camino hacia una forma de aprender matemáticas diferente a la que los maestros en formación han estado habituados.

La calidad de la formación es, desde nuestra perspectiva, fruto de la interacción entre el conocimiento sobre contenido matemático, y el conocimiento didáctico del contenido y esta interacción puede propiciarse especialmente cuando los futuros maestros tienen la oportunidad de enfrentarse a problemas que desafían su inteligencia, en contextos que invitan a la exploración y la especulación como medio para aprender ideas y procedimientos matemáticos que ellos mismos tendrán luego que desarrollar con sus estudiantes (Cai, 2003; Kilpatrick, Swafford y Findell, 2001). Como señalan Boavida, Delgado, Mendes y Brocardo (2018), citando a Kilpatrick, et al, (2001, p. 370), es esencial que los futuros maestros desarrollen "la capacidad de ver las posibilidades matemáticas en una tarea", junto con la capacidad de generar nuevas tareas que promuevan el razonamiento y resolución de problemas.

Como veremos a continuación, de las cuatro fases o formas de abordar la resolución de problemas, de Kilpatrick (1985), Osmosis, Memorización, Imitación, Cooperación y Reflexión, nuestro trabajo se centra en esta última, en la cual las habilidades para resolver problemas se adquieren de manera orientada a la acción y a través de la reflexión sobre enfoques para el planteamiento y la resolución de problemas. Es en esta fase donde, se produce lo que Bruder (2000) 1lama transferencia. Es en este momento cuando los resolutores exitosos transfieren, más fácilmente que otros, un procedimiento conocido de un contexto a otro, a veces incluso muy diferente. Reconocen más fácilmente el esquema común de un grupo de tareas similares.

\section{La experiencia en la Universidad de Huelva}

Como se ha señalado antes, en la formación de Maestros de Primaria, desde la perspectiva de la Didáctica de la Matemática, en la Universidad de Huelva, se articula en torno cuatro pilares. El primero, de carácter transversal, aunque ocupa el contenido de una de las cuatro asignaturas, 
es el planteamiento y resolución de problemas; los otros tres tienen su mirada en los aspectos de la educación matemática relacionados con los grandes bloques de contenidos matemáticos de la Educación Primaria: Números y operaciones; la construcción del lenguaje matemático, magnitudes y medida y tratamiento de la información, azar y probabilidad, y las formas, las figuras y sus propiedades. La materia que aborda el planteamiento y resolución de problema está situada en el primer curso. Bajo la denominación de Introducción a la Enseñanza y Aprendizaje de la Matemática en la Educación Primaria, se pretende provocar un choque respecto a la tradición en educación matemática que ha caracterizado la formación de quienes acceden nuestros centros. Como se ha comentado antes, somos conscientes de las escasas o nulas experiencias que los futuros maestros han tenido en relación con el planteamiento y resolución de problemas matemáticos; esta asignatura pretende ser una oportunidad de ver otra forma de hacer matemáticas, a través del planteamiento y resolución de problemas. Los futuros maestros adquieren un doble papel; por un lado, los situamos como resolutores, de forma que vivan la experiencia como aprendices; de otro, les llevamos a una reflexión metacognitiva sobre los procesos que realizamos y sus consecuencias de cara al aprendizaje de sus futuros alumnos. Pero en su papel de resolutores, como se ha ido comentando, no nos limitamos a que resuelvan problemas, más bien ponemos el énfasis en el proceso de transformación para lograr nuevos enunciados que modifique, los datos, el contexto e, incluso, parte de la estructura de los problemas. De esa manera conseguimos una comprensión profunda de los heurísticos (Liljedah et al., 2016) que trabajamos y de los procesos de pensamiento que nos llevan a la solución y una integración de las estructuras de conocimiento existentes (Kiliç, 2017).

Plantear problemas, además de ampliar en los futuros maestros su comprensión de las matemáticas y de potenciar su capacidad de explorar la naturaleza de los problemas en lugar de centrarse solo sus a soluciones (Stoyanova, 2003), beneficia a los futuros maestros de múltiples formas. En primer lugar, como hemos comentado, potencia la capacidad de comprender los procesos cognitivos, fomentado la metacognición (Mestre, 2002); en segundo lugar, les ayuda a comprender el papel que el planteamiento y resolución de problemas tiene en el currículo, y su relación con las competencias matemáticas que se promueven (Cai, Moyer, Wang, Hwang, Nie y Garber, 2012); y, por último, les ayuda a desarrollar y fortalecer sus habilidades de pensamiento crítico.

Nuestras estrategias de aprendizaje por transformación, para la elaboración de nuevos enunciados a partir de un problema dado, se basan en la categoría de Silver (1994), posterior a la solución, similar a la de situaciones estructuradas de Stoyanova (2003).

De todos los heurísticos que trabajamos en esta asignatura, hemos elegido uno que pensamos que ayuda mejor a comprender el trabajo que desarrollamos. No daremos nombre al heurístico, de momento. Dejaremos al lector que, a través de la comprensión de su funcionamiento, le de su propio nombre, aunque al final sí diremos cómo le llamamos nosotros. 
En lo que sigue procuraremos hacer ver diferentes acciones que se desarrollan en varias sesiones de clase. La primera de ellas podría comenzar con el siguiente enunciado:

"En un corral hay gallinas y conejos, alcanzamos a ver 13 animales y contabilizamos 42 patas, ¿cuántas gallinas y conejos hay?”

Cada vez que este enunciado forma parte de las primeras clases de la asignatura, los futuros maestros suelen abordarlo mediante un sistema de dos ecuaciones con dos incógnitas, pero cuando llegan a la solución les cuestiono sobre la viabilidad de utilizar esta potente herramienta algebraica en tercer curso de Educación Primaria, que es donde este problema es planteado en un libro de texto. Esta sencilla reflexión curricular les pone ante el reto de encontrar una forma de encontrar la solución, adecuada, para ese nivel educativo. A veces, algún estudiante ha aportado la solución que comentaré; cuando no es sí, les cuento cómo observé resolverlo a un niño de 9 años.

Este niño dibujó 13 círculos que representaban las 13 cabezas (todas iguales pues no sabía a que tipo de animal correspondían) y, a continuación, puso dos palitos en cada una de ellas (cada palito dijo que representaba a una patita, afirmando que cada animal tiene al menos dos). A continuación, contó las patatitas que ya ha había colocado (26) y las comparó con las que tendría que haber (42). La diferencia (42-26 = 16) sabía que había que colocarlas de dos en dos (aunque él no era consciente de ello, estaba transformando gallinas en conejos). El registro gráfico le aportó la solución al problema.

Varias reflexiones sobre esta solución son necesarias para comprender qué está debajo del razonamiento del niño. En primer lugar, no se nos debe pasar por alto la importancia del cambio de registro verbal a registro gráfico; en muchos casos, este procedimiento mejora nuestra capacidad de comprensión y de planificación. En segundo lugar, es necesario reflexionar acerca de las operaciones que están en juego, para comprobar que resulta un problema con requerimientos adecuados para el tercer curso de Primaria, donde lo hemos encontrado. En tercer lugar, analizado desde el punto de vista algebraico, lo que el niño ha hecho (naturalmente sin ser consciente de ello) es partir de una solución particular de la ecuación más sencilla $(x+y=13)$, en este caso que el número de conejos es cero. Después ha visto si se cumple la segunda condición (ha contado el número de patitas que habría), al comprobar que no eran suficientes, ha ajustado el resultado, transformando gallinas en conejos.

Llegados a este punto les pregunto a mis alumnos (futuros maestros) si creen que la estrategia podría funcionar tomando dos valores cualesquiera que sumen 13 y reproduciendo el procedimiento seguido por el niño. Para que vean que el proceso es independiente de los valores que tomen (salvo los valores que resuelven la ecuación, lo que nos hace toparnos con la solución sin que ello nos aporte nada), divido la clase en grupos, de forma que cada grupo lo haga con valores diferentes, y me aseguro, además, de que algunas de las combinaciones que sumen 13 lleven a una situación en la que el número de patitas asignadas en la primera fase del proceso sea superior al total (pasarse, en vez de quedarse corto), de 
forma que a la transformación de gallinas en conejos, que supone el quedarse corto, añadimos ahora transformar conejos en gallinas (quitando patitas sobrantes, de dos en dos).

En la fase de ajuste del problema suelen hacer una división por dos (añadir o quitar patitas de dos en dos), donde el dividendo es la diferencia entre las patitas puestas y las que tiene que haber, el divisor dos (número de patitas que quitamos cada vez) y el cociente es el número de animales que se transforman. A pesar de que se hacen explícitos los significados de estos valores, el valor del divisor tiene la particularidad de ser la diferencia entre 4 y 2 (número de patitas de cada tipo de animal) y también la mitad de 4. Esto, como veremos después, nos mostrará que este ejemplo no es transparente respecto de la relación entre 4 y 2 que necesitan utilizar. Para abordar esta cuestión les propongo que enuncien un problema similar cambiando solo el contexto. No sin dificultades, generan un contexto que les resulta familiar, relacionando ruedas de motocicletas y automóviles. El problema surge cuando, además de cambio de contexto, les solicito cambio de datos. Pueden elegir cualquier combinación lineal donde no aparezcan, a la vez, 2 y 4, precisamente para poder profundizar acerca del tipo de relación entre estos valores.

Es algo que les suele costar trabajo y, a veces, se hace necesario sugerir el contexto. Por ejemplo, una celebración donde hay un determinado número de comensales, que hemos de sentar alrededor de mesas de dos tamaños (que acogen a dos números diferentes de comensales). Tanto en esta transformación, como en la anterior, los futuros maestros no toman conciencia fácilmente de dos aspectos esenciales a la hora de enunciar un problema. El primero es que es preciso partir de lo que será la solución; el segundo es que algunos de los datos pueden ser aleatorios (en este caso, la capacidad de cada tipo de mesa y el total de mesas), pero que hay un dato que es dependiente de los anteriores, el número total de comensales. Estos dos aspectos es necesario trabajarlos cuando se entrena en formular problemas.

Imaginemos la siguiente formulación posible, en un contexto en el que se dispone de 13 mesas, con capacidad para 5 y 9 comensales, respectivamente, lo que nos llevaría a un total de (elijamos 10 de 5 y 3 de 9) 77 invitados:

"En una celebración se dispone de 13 mesas de 5 y 9 comensales, respectivamente, si hay que sentar a 77 invitados, ¿̇cuántas mesas de 5 y de 9 serán necesarias?"

Los futuros maestros abordan la resolución utilizando el heurístico que están aprendiendo. Toman dos números naturales que sumen 13, por ejemplo 6 y 7, y asumen que la solución fuera 6 mesas de 5 comensales, y 7 mesas de 9 comensales. Ello conduciría a un total de $30+63=93$ comensales. Como en este caso nos hemos pasado, averiguamos en cuanto, es decir restamos 77 de 93, obteniendo 16. En este punto ya han asumido que esa cantidad de exceso es preciso ajustarla a 0 , a través de la transformación de mesas del tamaño mayor a mesas del tamaño menor y para saber el número de mesas, saben que han de hacer una división. Es aquí cuando se desconciertan, pues la relación entre 4 y 
2 es preciso ahora determinar si era aditiva o multiplicativa. Algunos, de forma automática dividen por 2 el exceso, justificando que hay "dos tipos" de mesas, pero claro, no les funciona. Es preciso entonces ayudarles a reflexionar sobre el significado de transformar mesas de 9 en mesas de 5, "perdiendo" 4 comensales (la diferencia entre 9 y 5) cada vez. Es decir, es 4 el divisor por el que dividir el exceso (16), obteniendo el número de transformaciones. Es ahora cuando descubre la relación aditiva que les permite conocer el sentido del ajuste.

Es momento, cuando se ha llegado aquí, de hacer una reflexión sobre la estructura común a los problemas que se han ido enunciando y resolviendo. Se trata, en todos los casos de un sistema de dos ecuaciones con dos incógnitas de la forma:

$$
\begin{gathered}
x+y=a \\
m x+n y=b
\end{gathered}
$$

Con a, b, m y n números naturales.

La transformación que sigue va a afectar también a esta estructura. Les pedimos que enuncien un problema en el que m o n sean números enteros. Esta transformación les cuesta bastante más que las anteriores, a pesar de que el contexto más cercano lo tienen "delante de su nariz", por ejemplo, las pruebas tipo test, en los que las preguntas acertadas suman un valor positivo, y las erróneas un valor negativo. Por ejemplo:

"En un examen tipo test hay 50 preguntas, las que se responde correctamente son valoradas con 3 puntos; las que se responden de forma errónea, restan 1 punto. Una persona, que respondió a todas las preguntas, obtuvo 90 puntos, ¿̇cuántas preguntas contestó correcta y erróneamente?"

Al llegar aquí se han superado algunos obstáculos que hemos ido comentando:

a. Son capaces de cambiar de registro verbal a gráfico y algebraico.

b. Identificado el contexto, son capaces, de identificar los datos que son arbitrarios y los que son dependientes de los anteriores.

c. Saben utilizar el heurístico y conocen la relación entre los coeficientes m y n de la segunda ecuación que les permite obtener el divisor para el momento del ajuste.

Es justamente en este aspecto donde surge ahora el problema. Imaginemos que suponen que hubo 25 preguntas acertadas y 25 erróneas. Ello conduciría a un total de $75-25=50$ puntos. Dado que la puntuación del enunciado es de 90, la diferencia es $90-50=40$. Ahora, siguiendo el procedimiento 
que casi han mecanizado, buscarán el número por el que dividir 40 para obtener el ajuste. Casi siempre, cuando llegamos a este punto, intentan hacer la diferencia entre 3 y 1, los coeficientes m y n. Este proceder. No es correcto.

Desde mi punto de vista, el mayor enemigo del entrenamiento de heurísticos es justamente su aplicación mecánica. Hay dos aspectos que pueden verse ahora si no se ha hecho antes. En primer lugar, han de tomar conciencia de que el número que obtienen en las diferencias, en cada caso, (aquí sería $90-50=$ 40 ), sean cuales sean los valores con los que prueben, es siempre un múltiplo del cociente que tienen que buscar $(m-n)$, pues de otra manera no tendrían una división exacta; en segundo lugar, dado que la segunda ecuación ahora es $3 x-y=90$, el signo menos no ha de considerarse como resta, sino como parte del coeficiente $n$, es decir, la ecuación es, realmente $3 x+(-1) y=90$. Por tanto, $m-n=3-(-1)=4$. Esta consideración de los coeficientes, como números con signo, tiene importantes implicaciones en el aprendizaje significativo de los números enteros.

Es el momento de lanzar un nuevo reto. ¿Es posible enunciar un problema donde la primera ecuación sea de la forma $x-y=a$ ?, ¿cambiará esto en algo el funcionamiento de nuestro heurístico?

Dijimos al principio que le daríamos nombre al heurístico cuando ya supiéramos cómo era; quizás ha llegado el momento. Lo llamamos "ensayo-error controlado". A diferencia del ensayo-error aleatorio o incluso sistemático, que te lleva a la solución a través de múltiples pruebas, aquí nos basta una sola prueba y el correspondiente ajuste reflexivo. Pero, volvamos al reto, probablemente el más complejo de todo el proceso que llevamos descrito.

Un posible contexto es el de un ascensor de un bloque de viviendas que se ha averiado y repite una secuencia de subidas y bajadas que no puede controlar el usuario, por ejemplo:

"El ascensor de mi bloque, que tiene 60 plantas, se ha vuelto loco. Esta mañana lo tomé en la planta baja y tras subir un número de pisos que no llegué a contar, para bajar después otra cantidad de pisos, me dejó en la planta 5a. Sólo te puedo decir que, si hubiera realizado la misma subida 5 veces y la misma bajada 3 veces, me habría dejado en la planta 39. ¿Me puedes decir el número de pisos que sube y baja en su averia?"

La estructura algebraica de este problema es el siguiente sistema de ecuaciones:

$$
x-y=5
$$

$$
5 x-3 y=39
$$

Conviene hacer explícito que, desde nuestra perspectiva, el conocimiento de los maestros ha de ir más allá del conocimiento que movilizará en sus estudiantes; por ello, exigir a los futuros maestros 
el conocimiento de las herramientas algebraicas que les permitan comprender la estructura de los problemas y el funcionamiento de los heurísticos, no está reñido con la exigencia, también, de que su resolución se aborde, como lo hemos estado haciendo hasta ahora, con herramientas al alcance de los niños de Educación Primaria. Así que, resolvamos el problema anterior, como hemos hecho hasta ahora y comprobemos qué supone el cambio que se ha introducido.

Tomamos, para ello, dos números enteros, cuya diferencia sea 5, por ejemplo 9 y 4 . Imponemos la condición de la segunda ecuación, con lo que tenemos que 5×9-3×4=33; nos hemos “quedado cortos" en $39-33=6$.

Ahora toca interpretar qué significa, en este caso, "quedarse corto".

En los casos anteriores, por ejemplo, en el problema de las gallinas y conejos, suponía transformar gallinas en conejos, de forma que cada transformación me permitía "ajustar" dos patas; o en el ejemplo de los comensales, transformar mesas de 5 en mesas de 9, "ajustando", cada vez, 4 comensales.

En los casos anteriores esto había que hacerlo manteniendo fijo el valor de $\mathrm{x}+\mathrm{y}$, es decir, una vez obtenido el número de "ajustes", se realizaba un incremento en el valor de $\mathrm{x}$, que implicaba una disminución en el valor de y (o viceversa). Ahora hemos de dejar constante el valor de $x-y$, eso significa que el valor de ajuste que obtengamos ha de sumarse (o restarse, en caso de "pasarse") a minuendo y sustraendo. Esta es la esencia del cambio de estructura de la primera ecuación y ello implica que el valor que hasta ahora se obtenía dividiendo la "desviación" (en este caso 6), entre $\mathrm{m}$ - n, ahora ha de hacerse entre $m+n$; y es precisamente el valor que nos lleva a 3 (6:2), es decir, debemos incrementar en 3 tanto el número de subidas como el de bajadas, obteniendo como solución $x=9+3 ; y=4+3$.

Como hemos podido ir comprobando, el proceso de transformación y elaboración de nuevos enunciados que van modificando, datos, contexto o estructura en relación con el primero de los enunciados, nos va aportando una comprensión cada vez más profunda de la estructura común a todos los problemas y del funcionamiento y adaptación del heurístico, cuyo uso, en ningún momento se hace de forma mecánica.

Si recapitulamos, nuestro heurístico nos ha permitido plantear y resolver problemas cuya estructura algebraica es de la forma:

$$
\begin{gathered}
x \pm y=a \\
m x \pm n y=b
\end{gathered}
$$

Con a, b, m y n números enteros (hasta ahora, distintos de cero). Es fácil generalizar a números racionales.

Llegados a este punto, les pregunto a mis alumnos (futuros maestros) si el siguiente problema está incluido en la familia de problemas que acabamos de resolver: 
"El abuelo de Jorge tiene 8 veces más años que Jorge y la diferencia de edades el 49, ¿̇qué edad tiene cada uno?

Su estructura es:

$$
\begin{gathered}
x-y=49 \\
x=8 y(8 y-x=0)
\end{gathered}
$$

Por tanto, es de la forma señalada en el último sistema genérico, aunque su resolución requiere un poco de atención acerca de un aspecto que se ha podido pasar por alto en la reflexión inmediatamente anterior.

En la resolución del último problema enunciado, la diferencia obtenida en el "ajuste" era siempre positiva, pues elegíamos como minuendo el número mayor, tanto si nos "quedábamos cortos", como si nos "pasábamos". Pero veamos ahora qué puede ocurrir. Imaginemos que tomamos como candidatos $x=50 ; y=1$, que cumplen la primera ecuación. Si analizamos el cumplimiento de la segunda, tenemos $8 y-x=8-50=-42$. Esto puede provocar conflicto entre los estudiantes. Además, ahora $m+n$ (como correspondería a este esquema, de resta en las dos ecuaciones), toma el valor de 9 , cuando sabemos que los ajustes habrán de hacerse en módulos de 7 . Veamos por qué.

Si asignamos el símbolo $\otimes$ a la edad de Jorge, la edad del abuelo vendría representada por $\otimes \otimes \otimes \otimes \otimes \otimes \otimes \otimes$; por tanto, la diferencia entre las edades sería $\otimes \otimes \otimes \otimes \otimes \otimes \otimes$. Dado que esa diferencia es 49 , el valor de $\otimes$ ha de ser 7 . Esto justifica nuestra afirmación anterior, pero, además, nos devuelve al registro gráfico como punto de partida de la reelaboración del heurístico, cuando es necesario.

Con lo anterior, tomamos conciencia de que, en este caso, volvemos a m-n como valor del divisor para el ajuste, pero ¿qué hacemos con el resultado negativo de $8 y-x$ ?, pues en este caso, esa diferencia (con los valores que hemos tomado para $x$ e $y$ ) es $8-50=-42$. Basta considerar que $8 y-x=0$ es equivalente a $x-8 y=0$. Por tanto, nuestro ajuste lo haremos con el cociente (6), resultado de dividir 42:7. Seis años que hemos de detraer de la edad supuesta del abuelo, y añadir a la edad supuesta de Jorge.

\section{Coda}

El análisis con los futuros profesores, para comprender las características que han de tener los enunciados de problemas susceptibles de ser resueltos con el heurístico que hemos denominado "ensayo-error controlado", podría ir incluso un poco más allá. Podríamos, usando alguna herramienta de geometría dinámica, como Geogebra, analizar el significado del ajuste que realizamos, al considerar todos estos sistemas de ecuaciones como pares de rectas que se cortan, en los que la solución es encontrar el punto 
de corte. No abundaremos en este aspecto en este artículo, pero sí aprovecharemos la oportunidad para reflexionar acerca de la diferencia entre el conocimiento del profesor y el conocimiento que éste ha de desarrollar en sus estudiantes.

Argumentábamos en el segundo epígrafe de este artículo que, para que los maestros planteen y resuelvan problemas en su clase de Educación Primaria, en el sentido que hemos dejado traslucir en este trabajo, era preciso que su formación incidiera de forma explícita en este aspecto, concediendo una oportunidad de entender y hacer la matemática de forma diferente a como ellos la habían aprendido. Hay ideas desarrolladas en este trabajo, para trabajar con futuros maestros, que pueden tener su traslación inmediata al aula de Educación Primaria. El desarrollo de estrategias de resolución de problemas, como la que se ha desarrollado en el último epígrafe, o animar a los estudiantes en la búsqueda de diferentes enunciados en los que se modifiquen datos o contexto debería formar del trabajo de los niños; es una forma de potenciar su creatividad, de ver la importancia de los procesos frente a los resultados, de inmiscuirlos en actividades matemáticas auténticas (Singer et al., 2011) y desarrollar sus competencias matemáticas, entendidas como logros integradores de la comprensión conceptual, la fluidez procedimental, la adquisición de estrategias, el razonamiento adaptativo y la capacidad productiva (Boavida, Delgado, Mendes y Brocardo, 2018). El futuro maestro debe ir más allá. Debe tener la oportunidad de reflexionar acerca de los elementos de variación que hemos ido considerando en el epígrafe anterior, de las dificultades que cada tipo de enunciado o resolución puede tener para sus alumnos, de la forma de elegir la estructura para hacer transparente, en cada momento, una dimensión de variación frente a otras, de organizar la secuencia de resolución y enunciado de las variantes de un mismo problema atendiendo a la dimensión de variación que desea desarrollar en ese momento. Debe reflexionar, además, de los diferentes registros de representación que pueden ayudar a superar las dificultades que los estudiantes encontrarán en el proceso de comprensión, planificación, ejecución y análisis de la solución de un problema y debe tener los conocimientos de los fundamentos matemáticos inmersos en el heurístico que está tratando que aprendan sus alumnos.

La experiencia que se ha detallado en el apartado anterior se corresponde con el desarrollo de un heurístico, el que hemos denominado "ensayo-error controlado", pero de la misma forma trabajamos otros heurísticos con los futuros maestros a lo largo de la asignatura descrita. Trabajar marcha atrás, identificar submetas (dividir un problema en partes o resolver uno más sencillo), buscar patrones, generalizar, aprender a considerar toda la información (donde puede incluirse mirar desde otro punto de vista), como heurísticos más propios de la fase de planificación, junto con utilizar diferentes registros de representación u organizar la información (por ejemplo, en forma de una tabla), más frecuentes en la fase de comprensión. En esta asignatura, el desarrollo de la capacidad de plantear y resolver problemas, el análisis de su papel transversal en el currículo de Primaria y su relación con la adquisición de las competencias matemáticas, muestra un enfoque de "aprender y aprender a enseñar a plantear y resolver problemas”. En las otras asignaturas tres que completan la formación de maestros de educación primaria en la Universidad de Huelva, se complementa esa opción con "aprender y 
aprender a enseñar resolviendo problemas", donde no se pierden de vista los diferentes bloques de contenido de las matemáticas de la Educación Primaria.

\section{Referencias}

Boavida, A. M., Delgado, C., Mendes, F., y Brocardo, J. (2018). Challenging preservice teachers to produce varied mathematical problem solving strategies. Journal of the European Teacher Education Network, 13, 45-57.

Bruder, R. (2000). Akzentuierte Aufgaben und heuristische Erfahrungen. In W. Herget y L. Flade (Eds.), Mathematik lehren und lernen nach TIMSS. Anregungen für die Sekundarstufen (pp. 69-78). Berlin: Volk and Wissen.

Cai, J. (2003). What research tells us about teaching mathematics through problem solving. In F. Lester Jr. (Ed.). Teaching mathematics through problem solving: prekindergarten-grade 6 (241-254). Reston: NCTM.

Cai, J., Moyer, J. C., Wang, N., Hwang, S., Nie, B., y Garber, T. (2012). Mathematical problem posing as a measure of curricular effect on students'learning. Educational Studies in Mathematics, 83(1), 57-69.

Carrillo, J. y Contreras, L.C. (2020). Formación del profesorado de matemáticas en España. En H.D. García e Lacerda, D.S. Correa e M.V. Maltempi (Orgs.), Formaçao inicial de profesores de matemática em diversos países (pp. 123-140). Sao Paulo: Editora Livraria da Física.

Carrillo, J., Contreras, L. C., Climent, N., Montes, M. A., Escudero-Avila, D., y Flores-Medrano, E. (Coords.) (2016). Didáctica de las Matemáticas para Maestros de Educación Primaria. Madrid: Paraninfo.

Castro, E. (Ed.) (2001). Didáctica de la matemática en la Educación Primaria. Madrid: Síntesis.

Chamorro, C. (Coord.) (2003). Didáctica de las Matemáticas. Madrid: Pearson and Prentice Hall.

Christou, C., Mousoulides, N., Pittalis M., Pitta-Pantazi, D., y Sriraman, B. (2005). An empirical taxonomy of problem posing process. ZDM Mathematics Education, 37(3), 149-158.

Ellerton, N. (2013). Engaging pre-service middle-school teacher-education students in mathematical problem posing: Development of an active learning framework. Educational Studies in Mathematics, 83(1), 87-101.

Flores, P y Rico, L. (Coords.) (2015). Enseñanza y aprendizaje de las matemáticas en educación Primaria. Madrid: Pirámide. 
Godino, J. D. (Dir.) (2004). Didáctica de las matemáticas para maestros. Granada: Departamento de Didáctica de las Matemáticas, Universidad de Granada.

Hill, H. C., Rowan, B., y Ball, D. L. (2005). Effects of teachers' mathematical knowledge for teaching on student achievement. American Educational Research Journal, 42(2), 371-406.

INECSE (2003). PISA 2003.Pruebas de matemáticas y de solución de problemas. Madrid: INECSE.

Jäder, J., Lithner, J., y Sidenvall, J. (2019). Mathematical problem solving in textbooks from twelve countries. International Journal of Mathematical Education in Science and Technology (first on line), DOI: 10.1080/0020739X.2019.1656826

Karimi, S., Shahvarani, A., y Haghverdi, M. (2019). The role of problem-based Mathematics teaching according to the Kirkpatrick's Model on problem-solving performance of mathematics teachers. Journal for Educators, Teachers and Trainers, 10(1), 12-26.

Kiliç, Ç. (2013). Pre-service primary teachers' free problem posing performances in the context of fractions: An example from Turkey. The Asia Pacific Education Researcher, 22(4), 677-686.

Kiliç, Ç. (2017). A new problem-posing approach based on problem-solving strategy: Analyzing pre-service primary school teachers' performance. Educational Sciences: Theory and Practice, 17, 771-789.

Kilpatrick,J. (1985). A retrospective account of the past 25 years of research on teaching mathematical problem solving. In E. Silver (Ed.), Teaching and learning mathematical problem solving: Multiple research perspectives (pp. 1-15). Hillsdale, New Jersey: Lawrence Erlbaum.

Kilpatrick,J. (1987). Problem formulating: Where do good problem come from? En A. H. Schoenfeld (Ed.), Cognitive science and mathematics education (pp. 123-147). Hillsdale, NJ: Erlbaum.

Kilpatrick, J., Swafford, J., y Findell, B. (Eds). (2001). Adding It Up: Helping Children Learning Mathematics. Washington: National Academy Press.

Liljedah, P., Santos-Trigo, M., Malaspina, U., y Bruder, R. (2016). Problem Solving in Mathematics Education. Hamburgo: Springer.

Lowrie, T. (2002). Designing a framework for problem posing: Young children generating openended tasks. Contemporary Issues in Early Childhood, 3(3), 354-364. 
Luo, F. (2009). Evaluating the effectiveness and insights of pre-service elementary teachers' abilities to construct word problems for fraction multiplication. Journal of Mathematics Education, 2(1), 83-98.

Mestre, P. J. (2002). Probing adults' conceptual understanding and transfer of learning via problem posing. Applied Developmental Psychology, 23, 9-50.

National Council of Teachers of Mathematics. (2000). Principles and standards for school mathematics. Reston, VA: NCTM.

Polya, G. (1945). How to solve it. Princeton: Princeton University Press.

Putnam, R., y Borko, H. (2000). What do new views of knowledge and thinking have to say about research on teacher learning? Educational Researcher, 29, 4-15.

Segovia, I., y Rico, L. (Coords.) (2011). Matemáticas para maestros de Educación Primaria. Madrid: Pirámide.

Silver, E. A. (1994). On mathematical problem posing. For the Learning of Mathematics, 14(1), 19-28.

Singer, F. M., Ellerton, N., Cai, J. y Leung, E. C. K.(2011). Problem posing in mathematics learning and teaching: a research agenda. In B. Ubuz (Ed.). Proceedings of the 35 th Conference of the International Group for the Psychology of Mathematics Education, Vol. 1, pp. 137-166. Ankara, Turkey: PME.

Singer, M., y Voica, C. (2008). Extrapolating rules: How do children develop sequences? En O. Figueras, J. L. Cortina, S. Alatorr, y A. Mepúlveda (Eds.), Proceedings of the Joint Meeting of PME 32 and PMENA XXX (Vol. 4, pp. 256-263). Morelia, Mexico: Cinvestav-UMSWH.

Stanic, G., y Kilpatrick, J. (1988). Historical perspectives on problem solving in the mathematics curriculum. En R. Charles y E. A. Silver (Eds.), The teaching and assessing of mathematical problem solving: Research agenda for mathematics education series (Vol. 3, pp. 1-22). Reston, Virginia: NCTM.

Stoyanova, E. (2003). Extending students' understanding of mathematics via problem posing. The Australian Mathematics Teacher, 59(2), 32-40.

Tichá, M., y Hošpesová, A. (2009). Problem posing and development of pedagogical content knowledge in preservice teacher training. En V. Durand-Guerrier, S. Soury-Lavergne, y F. Arzarello (Eds.), Proceedings of the ERME 6 (pp. 1941-1950). Lyon, Francia: INRP.

Verschaffel, L., Greer, B., y De Corte, E. (2000). Making sense of word problems. Lisse, The Netherlands: Swets and Zeitlinger. 
Zimmerman, B. J. (2000). Attainment of self-regulation: A social cognitive perspective. En M. Boekaerts, P. Pintrich, y M. Zeidner (Eds.), Handbook of self-regulation (Chap. 2). San Diego, CA: Academic Press. 